\title{
Outcomes of Dilation of Recalcitrant Pancreatic Strictures Using a Wire-Guided Cystotome
}

\author{
Sridhar Sundaram, Dhaval Choksi, Aditya Kale, Suprabhat Giri, Biswaranjan Patra, Shobna Bhatia and Akash Shukla \\ Department of Gastroenterology, Seth GS Medical College and King Edward Memorial Hospital, Mumbai, India
}

Background/Aims: Pancreatic strictures in chronic pancreatitis are treated using endoscopic retrograde cholangiopancreatography (ERCP) with plastic stent placement. The management of recalcitrant strictures remains a challenge, with the use of a Soehendra stent retriever or a needle knife described in case reports. Here, we discuss our experience with dilation of dominant pancreatic strictures with a 6-Fr cystotome.

Methods: A retrospective review of an endoscopy database was performed to search for patients with pancreatic strictures recalcitrant to conventional methods of dilation in which a cystotome was used. Technical success was defined as the successful dilation of the stricture with plastic stent placement. Functional success was defined as substantial pain relief or resolution of pancreatic fistulae.

Results: Ten patients (mean age, 30.8 years) underwent dilation of a dominant pancreatic stricture secondary to chronic pancreatitis, with a 6-Fr cystotome. Seven patients presented with pain. Three patients had pancreatic fistulae (two had pancreatic ascites and one had a pancreaticopleural fistula). The median stricture length was $10 \mathrm{~mm}$ (range, 5-25 mm). The head of the pancreas was the most common location of the stricture (60\%). Technical and functional success was achieved in all patients. One patient had self-limiting bleeding, whereas another patient developed mild post-ERCP pancreatitis.

Conclusions: The use of a 6-Fr cystotome (diathermy catheter) can be an alternative method for dilation of recalcitrant pancreatic strictures after the failure of conventional modalities. Clin Endosc 2021;54:903-908

Key Words: Dilation; Endoscopic retrograde cholangiopancreatography; Pancreatitis; Stents

\section{INTRODUCTION}

Chronic pancreatitis is characterized by inflammation and fibrosis with loss of acinar cells and islet cells of the pancreas. The changes in the pancreas include morphological changes in pancreatic ducts with irregularity, stricturing, dilatation, and formation of pancreatic ductal calculi with intraparenchymal small-duct calcifications. Pain in chronic pancreatitis may be

Received: November 9, 2020 Revised: January 2, 2021

Accepted: January 5, 2021

Correspondence: Sridhar Sundaram

Department of Gastroenterology, Seth GS Medical College and King Edward Memorial Hospital, 9th Floor, MS Building, Acharya Donde Marg, Parel, Mumbai 400012, India

Tel: +91-9860096846, Fax: +91-022-24177585, E-mail: drsridharsundaram@ gmail.com

ORCID: https://orcid.org/0000-0002-2946-8534

(c) This is an Open Access article distributed under the terms of the Creative Commons Attribution Non-Commercial License (http://creativecommons.org/ licenses/by-nc/3.0) which permits unrestricted non-commercial use, distribution, and reproduction in any medium, provided the original work is properly cited. related to obstruction secondary to strictures, and is known to respond to endoscopic therapy for pancreatic ductal strictures in $70-80 \%$ of the cases. ${ }^{1}$ In a previous study on extracorporeal shock wave lithotripsy of pancreatic calculi, the incidence of strictures was found to be $18 \%$ in patients with chronic pancreatitis. ${ }^{2}$ Pancreatic strictures can be classified as dominant or non-dominant. Dominant pancreatic ductal strictures with high-grade narrowing have been defined as the presence of at least one of the following: (1) upstream dilatation of the main pancreatic duct (MPD) $\geq 6 \mathrm{~mm}$, (2) failure of contrast to flow alongside the stricture or a 6-Fr nasopancreatic tube (NPT), and (3) presence of pain during continuous perfusion of normal saline through an NPT for $24 \mathrm{hr}^{3}$ MPD strictures can be secondary to benign or malignant etiologies. Endoscopic therapy is reserved for dominant strictures in benign pathologies.

The European Society of Gastrointestinal Endoscopy guidelines for endotherapy in chronic pancreatitis recommend placement of a single 10-Fr stent for pain relief in patients with dominant pancreatic strictures, uninterrupted for 1 year and 
with stent exchange(s) on demand. ${ }^{4}$ Although stent placement may lead to immediate pain relief in $80-90 \%$ of patients, long-term relief is observed in $67.5 \%$, as reported in a recent meta-analysis of nine studies with $>500$ patients. ${ }^{5,6}$ Refractory strictures are symptomatic dominant strictures that relapse or persist 1 year after single-stent placement. ${ }^{4}$ Dilation may be required for stent placement; however, some recalcitrant strictures may be extremely tight owing to the dense desmoplastic process, and hence resistant to dilation with conventional methods using bougies and balloons. ${ }^{3}$ Wire-guided diathermy catheters, such as cystotomes, are primarily used for dilation of the tract during endoscopic cystogastrostomy. When conventional methods of dilation fail, a wire-guided cystotome can be used for dilation of pancreatic strictures. This was first described by Kim et al. in 2011 in two patients with chronic pancreatitis with difficult-to-dilate strictures. ${ }^{8}$ They described both retrograde dilation using endoscopic retrograde cholangiopancreatography (ERCP) and antegrade dilation using endoscopic ultrasound-guided access to the pancreatic duct. Here, we describe our experience with dilation of recalcitrant pancreatic strictures using a wire-guided cystotome.

\section{MATERIALS AND METHODS}

A retrospective review of the endoscopy database of a tertiary care referral center was performed. The study was approved by the institutional ethics committee as a retrospective study (EC/16470). A total of 1,521 ERCP procedures were performed at our tertiary care referral center between August 2018 and March 2020. Among these cases, 133 patients required pancreatic duct drainage for various indications (pancreatic stricture, stones, pancreatic fistulae, or minor papilla sphincterotomy). Pancreatic stricture was the indication for ERCP in 57 patients. Of these, deep guidewire cannulation of the MPD was achieved in 54 (94.7\%) patients. Patients with failed dilation of pancreatic strictures with conventional methods, such as passage of a sphincterotome or a bougie dilator, were planned for dilation using a cystotome. Ten patients were candidates for dilation using a wire-guided cystotome. All patients underwent magnetic resonance cholangiopancreatography of the abdomen for planning the ERCP. Only patients with a single symptomatic dominant stricture of benign etiology were considered for the intervention.

All ERCP procedures were performed with the patient under monitored anesthesia care and total intravenous anesthesia with propofol, in the left lateral or supine position. ERCP was performed by endoscopists with experience of $>500$ ERCP procedures and with proficiency in ERCP procedures with grade 3 difficulty. ERCP was performed using an Olympus TJF Q180V (Olympus, Tokyo, Japan) sideview duodenoscope. Cannulation was performed using Clever Cut $3 \mathrm{~V}^{\mathrm{TM}}$ sphincterotomes (Olympus), and a Visiglide ${ }^{\mathrm{TM}}$ 0.025-in guidewire (Olympus) was used for manipulation. Pancreatography was performed to delineate the stricture. After guidewire passage, dilation was initially attempted using a Hurricane balloon dilator (Boston Scientific, Marlborough, MA, USA) and a Soehendra biliary dilation catheter (SBDC) (Cook Medical LLC, Bloomington, IN, USA; size, 6 Fr). When dilation with the above method failed, a subsequent dilation was performed with a 6-Fr wire-guided diathermy catheter with a metal electrocautery tip (Cysto-Gastro-Set; ENDO-FLEX[Medi-Globe], Rohrdorf, Germany) (Fig. 1). Endocut I (effect 2, cut duration 3, cut interval 3) current (ERBE Vio200; Erbe Elektromedicin Gmbh, Tübingen, Germany), as used for sphincterotomy (monopolar), was applied to ablate the stricture. Current was applied once the diathermy catheter was already abutting the stricture, with gentle persistent pressure until the length of the stricture was passed. Further dilation was attempted using an SBDC (7 Fr) after dilation with a cystotome. Thereafter, either

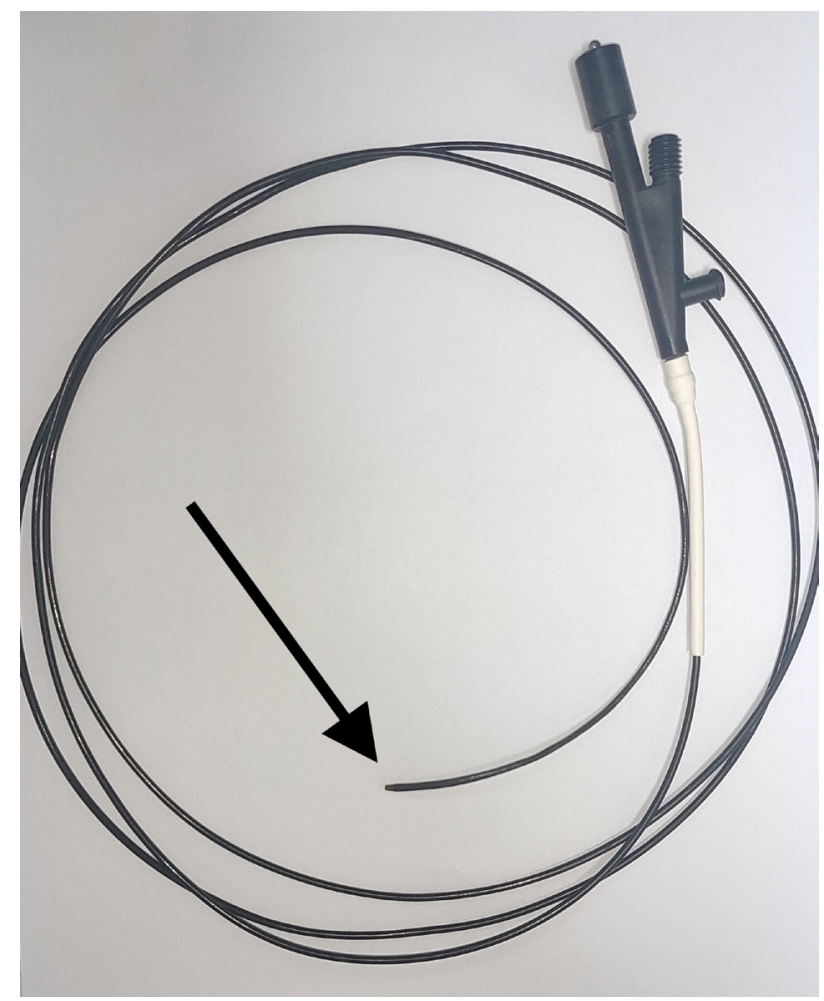

Fig. 1. A 6-Fr monopolar cystotome used for dilation. The arrow points to the metal tip. 
a 5- or 7-Fr stent with a single pigtail, depending on the extent of dilation, was placed across the stricture.

Technical success was defined as the placement of a stent with drainage of contrast in the dilated pancreatic duct beyond the stricture. Functional success was defined as substantial pain relief after stent placement, determined as a reduction in pain duration and intensity by at least 3 points on the visual analog scale (VAS). Pain was evaluated before and 2 weeks after the procedure to assess the functional success. On follow-up at 3 months, the patients were reassessed for pain. In three patients who had a pancreatic fistula with chronic pancreatitis and a tight stricture, resolution of the fistula was the indicator of functional success. At the 3-month follow-up, patients who had a fistula were assessed for pain and persistence of the fistula. Adverse events were graded according to severity, as defined in the American Society of Gastrointestinal Endoscopy guidelines.

\section{RESULTS}

\section{Demographics}

Of the 10 patients, 8 were men and 2 were women. The mean age of the patients was 30.8 years (range, 6-60 years). The underlying cause of the stricture was chronic pancreatitis in all patients. The etiology of chronic pancreatitis was alcohol related (50\%), abnormal pancreaticobiliary ductal union $(20 \%)$, or idiopathic $(30 \%)$. All patients were classified as having severe chronic pancreatitis according to the Cambridge classification. Of the 10 patients, 3 had a pancreatic fistula (2 had a pancreaticopleural fistula and 1 had pancreatic ascites). The location of the stricture was the head of the pancreas in six patients, the neck in two patients, and the body in two patients (Figs 2A-C, 3, and 4). One patient had a pancreas divisum with the stricture just beyond the opening of the minor papilla in the region of the head of the pancreas. The median

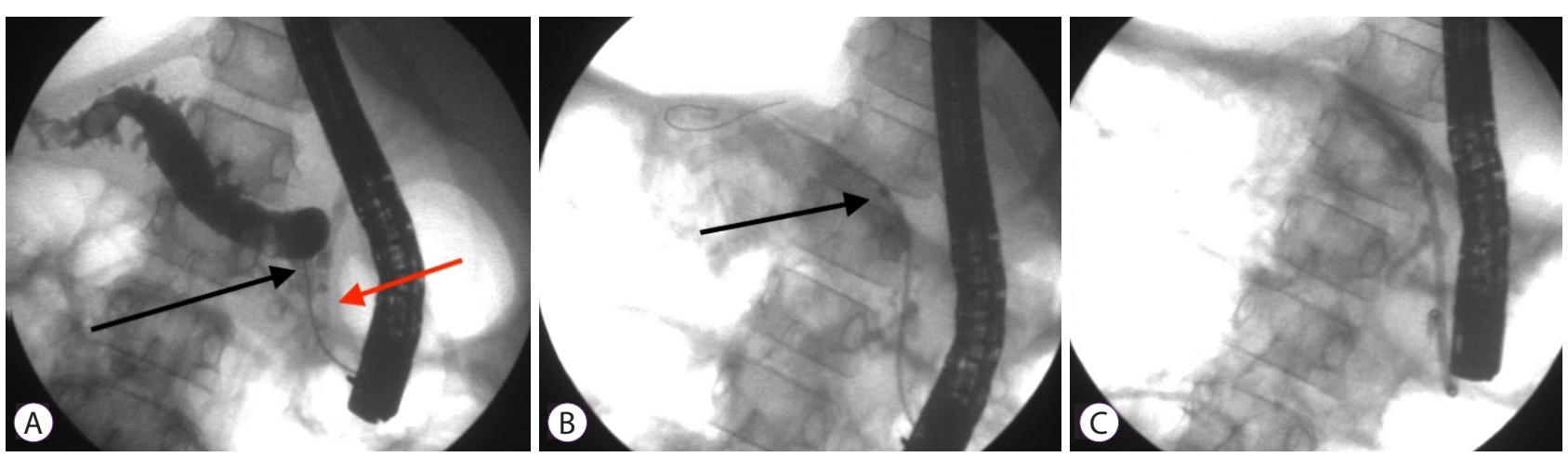

Fig. 2. (A) Stricture at the neck of the pancreas (black arrow) with abnormal pancreaticobiliary ductal union Komi type IIA with a long common channel (red arrow), with the cystotome in the region of the head. (B) Cystotome passed across the stricture (arrow), with evident drainage of contrast. (C) Stent placed with complete drainage of contrast.

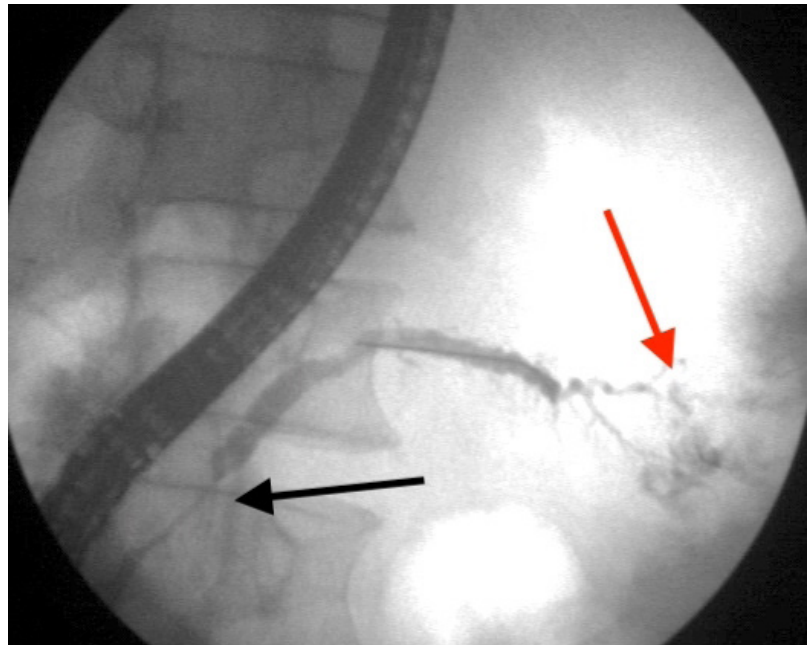

Fig. 3. Stricture in region of the head (black arrow) with leak from the tail of the pancreas (red arrow) leading to a pancreaticopleural fistula.

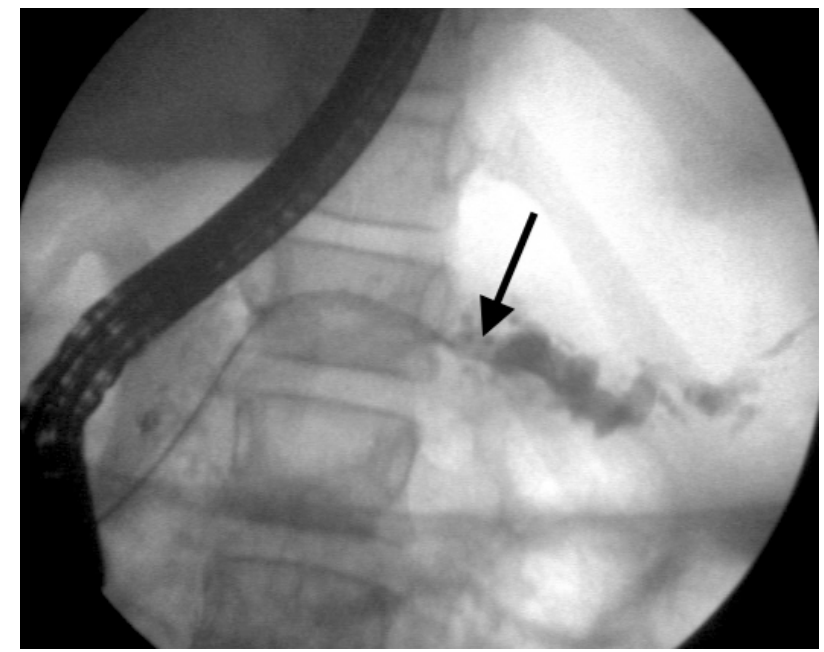

Fig. 4. Stricture in the proximal body of the pancreas (arrow) with a distally dilated pancreatic duct. 
length of the MPD strictures was $10 \mathrm{~mm}$ (range, 5-25 mm).

\section{Outcomes}

Dilation was technically successful in all patients (100\%), with successful stent placement in all cases. The median duration of cautery was $6 \mathrm{~s}$ (range, 3-20 sec). In two patients, further dilation with a 7-Fr SBDC was not feasible, and 5-Fr plastic stents were placed across the stricture. A 7-Fr single-pigtail plastic stent was placed in all other patients. Functional success was also achieved in all cases, with substantial pain relief in patients with dominant strictures causing pain (7/7 patients, $100 \%)$. All patients with pancreatic internal fistulae had a successful resolution (3/3 patients, 100\%) during the next 1 week. On follow-up at 3 months, all patients had substantial pain relief. In addition, none of the patients had a recurrence of the fistula.

\section{Adverse events}

A procedure-related adverse event was observed in one patient who had self-limiting bleeding after dilation with the cystotome, which spontaneously resolved after stent placement. One patient developed mild pancreatitis after ERCP, which resolved with conservative therapy. No other complications were observed. A summary of the results is provided in Table 1.

\section{DISCUSSION}

In this study, we evaluated the use of a 6-Fr cystotome for dilation of recalcitrant pancreatic duct strictures. Technical success was achieved in all patients. Conventional approaches include the use of balloons and bougies such as SBDC for dilation of strictures in the biliary and pancreatic ducts, followed by stent placement. Stents placed for strictures in painful chronic pancreatitis are associated with substantial short-and long-term benefits with respect to pain reduction. ${ }^{4}$ A recent series investigated the use of multiple stents in the setting of chronic pancreatitis with dominant strictures in the pancreatic head, with $75 \%$ of the patients remaining asymptomatic at a median follow-up of 9.5 years after the removal of the stents. Thus, dilation with stent placement is an important modality in the treatment of pain in patients with chronic pancreatitis with dominant strictures, even in the long term.

In the event of failed dilation with a balloon or bougie, the

Table 1. Summary of Results

\begin{tabular}{|c|c|}
\hline Characteristics & $n=10$ \\
\hline Mean age & 30.8 yr (range, 6-60 yr) \\
\hline Male:Female & $8: 2$ \\
\hline Underlying cause of stricture & Chronic pancreatitis \\
\hline Etiology of chronic pancreatitis & $\begin{array}{l}\text { Alcoholic- } 5 \\
\text { Abnormal pancreaticobiliary ductal union- } 2 \\
\text { Idiopathic- } 3\end{array}$ \\
\hline Presentation & $\begin{array}{l}\text { Pain- } 70 \% \\
\text { Pancreatico-pleural fistula- 20\% (leak seen from tail of pancreas) } \\
\text { Pancreatic ascites- 10\% (leak seen from body of pancreas) }\end{array}$ \\
\hline Location of stricture & $\begin{array}{l}\text { Head- } 60 \% \\
\text { Neck- } 20 \% \\
\text { Body- } 20 \%\end{array}$ \\
\hline Length of stricture & Median $10 \mathrm{~mm}$ (range, $5-25 \mathrm{~mm}$ ) \\
\hline Duration of cautery & Median 6 sec (range, 3-20 sec) \\
\hline Technical success & $10 / 10(100 \%)$ \\
\hline Functional success & $\begin{array}{l}100 \% \\
\text { (including resolution of pancreatic fistulae in all patients) }\end{array}$ \\
\hline Mean VAS prior to procedure & $6.57+1.7$ \\
\hline Mean VAS after stent placement & $2+1.15$ \\
\hline Complications & $\begin{array}{l}1 \text { patient developed self-limiting bleeding } \\
1 \text { patient developed mild post ERCP pancreatitis }\end{array}$ \\
\hline
\end{tabular}

ERCP, endoscopic retrograde cholangiopancreatography; VAS, visual analog scale. 
Soehendra stent retriever device has been used for dilation in a few case series. ${ }^{10,11}$ The stent retriever acts as a screw drill for dilation across the stricture. However, in some cases, the tip could not engage strictures that are extremely narrow, hence leading to the failure of these devices.

Non-coaxial techniques, such as the use of a precut sphincterotome, have been described for dilation of strictures. ${ }^{12}$ These techniques can only be used for strictures in the region of the pancreatic head. In addition, there is an increased risk of perforation, pancreatitis, and bleeding due to the non-coaxial nature of the cutting wire. Wire-guided snares can also be used with electrocautery for stricture dilation. However, the cutting wire is largely uncontrolled and non-coaxial despite passage over a wire in a given situation, thus increasing the risk of complications. ${ }^{13}$

Two previous studies have examined the use of a 6-Fr diathermy catheter for the dilation of tight biliary and pancreatic strictures. In the study by Kawakami et al., 16 patients with tight biliary strictures and 6 patients with tight pancreatic strictures with a history of failed dilation using Hurricane balloons, SBDCs, and Soehendra stent retrievers underwent dilation using a 6-Fr diathermy catheter. ${ }^{14}$ Stricture dilation was achieved in all patients. Two patients developed complications, in the form of hemobilia in one patient after biliary dilation and post-ERCP pancreatitis in another patient after pancreatic stricture dilation. In another study by Puri et al., 19 patients had tight biliary strictures and 6 had tight pancreatic strictures treated with dilation with a 6-Fr cystotome, with technical success achieved in all patients. ${ }^{15}$ In both series, only pancreatic strictures located in the head region were dilated using the cystotome. Clinical parameters associated with pain relief were not evaluated as an outcome in either study. Functional success in the form of substantial pain relief based on VAS scores was achieved in all patients included in our study. Three patients with internal pancreatic fistulae were also included in our study, all of whom showed improvement after the procedure. Both previous studies included only patients with strictures in the head of the pancreas, whereas our study included patients with strictures in the neck and body of the pancreas. Moreover, whereas the previous studies included only patients aged $>18$ years, our study included three patients aged $<18$ years.

Placement of a plastic stent for pancreatic strictures is associated with worsening of pancreatic pain in $6.2 \%$ of cases. ${ }^{16}$ However, most cases spontaneously resolve. Severe pancreatitis can also occur. Post-sphincterotomy bleeding is seen in 1.5\% of cases and sepsis in $2.6 \%$ after stent placement in the pancreatic duct. In our series, one patient had self-limiting bleeding, which occurred after dilation with a cystotome. No active intervention was required. One patient reported worsening of pancreatic pain immediately after the procedure; however, a substantial reduction in pain intensity and severity occurred

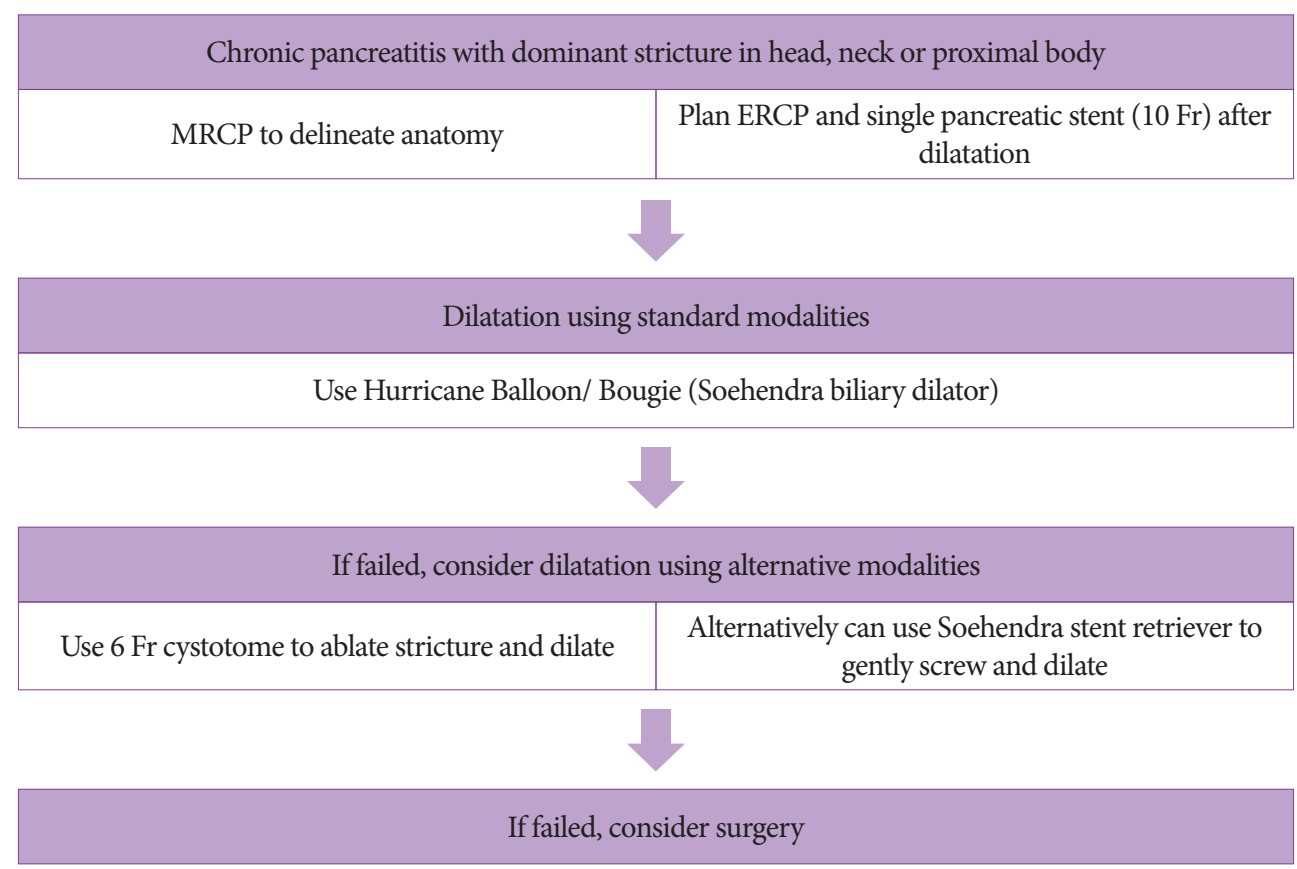

Fig. 5. Algorithm proposal for dilation of dominant pancreatic strictures. ERCP, endoscopic retrograde cholangiopancreatography; MRCP, magnetic resonance cholangiopancreatography. 
over 2 weeks, as compared with the pre-procedure status.

The limitations of our study include its retrospective nature, the small number of patients, and the non-randomized design with data from a single center. However, considering the rarity of recalcitrant single-dominant pancreatic strictures, no largescale data are available. In our study, improvement in clinical parameters as documented using VAS scores and resolution of fistulae validate the use of diathermy catheters for dilation of pancreatic strictures. In conclusion, the use of a 6-Fr cystotome for dilation of recalcitrant pancreatic strictures is an alternative method for patients in whom conventional modalities using bougies and balloons are not effective. Larger comparative studies are needed to establish the safety and efficacy of this modality in recalcitrant pancreatic strictures. In settings with failed dilation with balloon and bougies, diathermy catheters can be the next step in the algorithm of pancreatic stricture dilation before stent placement (Fig. 5).

\section{Conflicts of Interest}

The authors have no potential conflicts of interest.

Funding
None.

Author Contributions

Conceptualization: Sridhar Sundaram, Dhaval Choksi

Data curation: SS, DC, Suprabhat Giri, Biswaranjan Patra

Formal analysis: SS, Aditya Kale, SG, BP

Funding acquisition: SS, BP

Investigation: SS, AK, Akash Shukla, Shobna Bhatia

Methodology: SS, DC, AK, SG, BP, AS, SB

Project administration: SS, AK, AS, SB

Resources: SS, AK, AS, SB

Software: SG, BP

Supervision: SS, DC

Validation: AK, SG

Visualization: SS, DC, SG

Writing-original draft: SS

Writing-review\&editing: SS, DC, AK, SG, BP, AS, SB

ORCID

Sridhar Sundaram:

Dhaval Choksi:

Aditya Kale:

Suprabhat Giri:

Biswaranjan Patra:
Shobna Bhatia:

Akash Shukla:

https://orcid.org/0000-0001-6132-0356

https://orcid.org/0000-0001-7718-9452

\section{REFERENCES}

1. Godil A, Chen YK. Endoscopic management of benign pancreatic disease. Pancreas 2000;20:1-13.

2. Tandan M, Reddy DN, Santosh D, et al. Extracorporeal shock wave lithotripsy and endotherapy for pancreatic calculi-a large single center experience. Indian J Gastroenterol 2010;29:143-148.

3. Delhaye M, Matos C, Devière J. Endoscopic management of chronic pancreatitis. Gastrointest Endosc Clin N Am 2003;13:717-742.

4. Dumonceau JM, Delhaye M, Tringali A, et al. Endoscopic treatment of chronic pancreatitis: European Society of Gastrointestinal Endoscopy (ESGE) guideline - updated August 2018. Endoscopy 2019;51:179-193.

5. Seicean A, Vultur S. Endoscopic therapy in chronic pancreatitis: current perspectives. Clin Exp Gastroenterol 2015;8:1-11.

6. Jafri M, Sachdev A, Sadiq J, et al. Efficacy of endotherapy in the treatment of pain associated with chronic pancreatitis: a systematic review and meta-analysis. JOP 2017;18:125-132.

7. Saftoiu A, Vilmann A, Vilmann P. Endoscopic ultrasound-guided drainage of pancreatic pseudocysts. Endosc Ultrasound 2015;4:319-323.

8. Kim E, Aouattah T, Deprez PH. Successful retrograde and antegrade passage of narrow and complete pancreatic strictures using a cystogastrostome. Endoscopy 2011;43(Suppl 2):E340-E341.

9. Tringali A, Bove V, Vadalà di Prampero SF, et al. Long-term follow-up after multiple plastic stenting for refractory pancreatic duct strictures in chronic pancreatitis. Endoscopy 2019;51:930-935.

10. Faigel DO, Ginsberg GG, Kochman ML. Innovative use of the Soehendra stent retriever for biliary stricture recannalization. Gastrointest Endosc 1996;44:635.

11. Baron TH, Morgan DE. Dilation of a difficult benign pancreatic duct stricture using the Soehendra stent extractor. Gastrointest Endosc 1997;46:178-180.

12. Kawamoto H, Ishida E, Ogawa T, et al. Dissection of a refractory pancreatic-duct stricture by using a pre-cutting needle-knife. Gastrointest Endosc 2006;63:190-192.

13. Itoi T, Sofuni A, Itokawa F, et al. Transpapillary incision of refractory circumscript pancreatic duct stricture using wire-guided snare forceps. World J Gastroenterol 2010;16:1541-1544.

14. Kawakami H, Kuwatani M, Kawakubo K, et al. Transpapillary dilation of refractory severe biliary stricture or main pancreatic duct by using a wire-guided diathermic dilator (with video). Gastrointest Endosc 2014;79:338-343.

15. Puri R, Bhatia S, Bansal RK, Sud R. Endoscopic management of difficult benign biliary and pancreatic strictures using a wire-guided cystotome: experience with 25 cases. Endosc Int Open 2018;6:E797-E800.

16. Farnbacher MJ, Mühldorfer S, Wehler M, Fischer B, Hahn EG, Schneider HT. Interventional endoscopic therapy in chronic pancreatitis including temporary stenting: a definitive treatment? Scand J Gastroenterol 2006;41:111-117. 